\title{
Requisitos para o Desenvolvimento de Objetos de Aprendizagem Reutilizáveis para Dispositivos Móveis
}

\author{
João Roberto Ursino da Cruz ${ }^{1,2}$, Ana Maria Monteiro ${ }^{1}$ \\ ${ }^{1}$ UNIFACCAMP - Centro Universitário de Campo Limpo \\ Campo Limpo Paulista - SP - CEP 13231-2030 \\ ${ }^{2}$ UNISA - Universidade de Santo Amaro \\ Departamento de TI - Campus II - São Paulo - SP - 04743-030 \\ jrcruz9@gmail.com, anammont.aluegmail.com
}

\begin{abstract}
Among the phenomena that have been changing teaching methods, we highlight Distance Learning and, especially, mobile learning, a teaching modality that uses mobile devices as a learning tool. The apps used for this teaching mode are called Learning Objects Mobile (LOM). However, one of the factors that ultimately discourage teachers and researchers to adopt these LOM's as a tool in their disciplines is that the vast majority are developed for a specific content. This paper presents a requirements statement for the development of reusable mobile learning objects and based on these requirements we report the development of a test application.
\end{abstract}

Resumo. Dentre os fenômenos que vem mudando as formas de ensino destaca-se o Ensino à Distância e, em especial, mobile learning, modalidade de ensino, que utiliza os dispositivos móveis como ferramenta de aprendizagem. Os aplicativos utilizados para esta modalidade de ensino são chamados de Objeto de Aprendizagem Móvel (OAM). No entanto, um dos fatores, que acabam por desmotivar professores e pesquisadores a adotarem estes OAMs como ferramenta em suas disciplinas é que a grande maioria é desenvolvida para um conteúdo específico. Este artigo apresenta uma declaração de requisitos para o desenvolvimento de objetos de aprendizagem móvel reutilizáveis, e com base nestes requisitos, é apresentado no artigo o desenvolvimento de uma aplicação teste.

\section{Introdução}

O uso de dispositivos móveis em ambientes de aprendizagem, assim como em outros contextos, vem crescendo exponencialmente a cada ano. $\mathrm{O}$ estímulo ao desenvolvimento e novas TICs ligadas a M-Learning (Mobile Learning), deve-se aos estudos promovidos pelas instituições de ensino e a facilidade de acesso a dispositivos móveis por parte de professores e alunos, segundo Brasil et al. (2018).

Segundo o CGI.br (Comitê Gestor da Internet no Brasil), em 2016, o percentual dos alunos da $5^{\text {a }}$ a $9^{\text {a }}$ série das escolas públicas e privadas, que utilizam o celular para estudar, já ultrapassa os $52 \%$. O mesmo estudo, ainda aponta, que quanto maior o nível escolar, maior o número de alunos que utilizam estes dispositivos para realizar suas atividades. 
VIII Congresso Brasileiro de Informática na Educação (CBIE 2019)

Anais do XXX Simpósio Brasileiro de Informática na Educação (SBIE 2019)

A definição de OAMR (Objeto de Aprendizagem Móvel Reutilizável) foi cunhada a partir de várias outras definições, originando-se em Wiley (2000), que define o que são OAs (Objetos de Aprendizagem), passando por Pereira, Porto \& Melo (2003), que acrescentam aos OAs o conceito de reutilização de componentes e contextos (OAR - Objetos de Aprendizagem Reutilizáveis) e por Silva (2012), que destaca, que a criação de OAs para ambientes ubíquos escolares se diferencia dos demais não só pelo uso de dispositivos móveis, mas pela aplicação do que ele definiu como "sensibilidade de contexto educacional", o que posteriormente foi denominado de OAM (Objeto de Aprendizagem Móvel).

A partir destas definições, da análise literária sobre o tema, em particular do trabalho de Cruz (2017) e dos artigos que foram utilizados referência para este estudo, este artigo apresenta uma declaração com 10 requisitos para o desenvolvimento de OAMR, que serão apresentados na próxima seção e avaliados nas seções posteriores.

\section{Requisitos}

Para a definição dos requisitos de desenvolvimento do OAMR, inicialmente foi realizada uma pesquisa nos anais de eventos de tecnologia da educação, buscando por artigos de m-learning que detalhassem o desenvolvimento de aplicações mobile para este fim, destacando-se na pesquisa 37 publicações do CBIE e SBIE dos últimos 5 anos.

Posteriormente, usando o conceito de requisitos de sistema, apresentados por Sommerville (2011), e a análise feita nestes 37 artigos, foi criada uma compilação de 10 requisitos recomendados para serem utilizados por professores, pesquisadores e desenvolvedores em seus projetos de OAMRs. Os requisitos recomendados são:

1. Analisar e definir todos os paradigmas educacionais ligados ao OAMR: Antes de iniciar o processo de criação de um OAMR a equipe de desenvolvimento deve analisar todos os paradigmas que serão permeados pelas potenciais atividades pedagógicas, e o modo como estes otimizarão os processos de aprendizagem.

A definição destes paradigmas antes do início do processo de desenvolvimento pode auxiliar não só os professores na seleção das melhores atividades pedagógicas, mas também, os desenvolvedores no reuso das funcionalidades que suportarão o OAMR.

2. Possibilitar um fácil acesso de professores e alunos ao OAMR: $O$ conceito de acesso pregado neste requisito não se refere apenas a política de segurança, mas também ao acesso da aplicação em condições adversas ao ambiente escolar, como por exemplo, em momentos em que não haja uma conexão estável, ou quando o aluno ou o professor não possuam um dispositivo móvel.

Este requisito sugere que a equipe de desenvolvimento deve analisar funcionalidades que permitam que tanto professor quanto aluno possam utilizar a aplicação offline, ou em outras plataformas, como uma plataforma web, por exemplo, sem que haja prejuízo acadêmico na indisponibilidade de uma conexão.

3. Possuir ferramentas que estimulem a usabilidade por parte de alunos de faixas etárias diferentes: $O$ desenvolvimento de funcionalidades que permitam ao professor customizar não apenas o conteúdo didático, mas também, a interface e a linguagem usada nos feedbacks permitirão a reutilização destes OAMR. 
VIII Congresso Brasileiro de Informática na Educação (CBIE 2019)

Anais do XXX Simpósio Brasileiro de Informática na Educação (SBIE 2019)

Ao customizar as atividades pedagógicas considerando as faixas etárias e outras características de suas turmas, o professor cria a empatia dos alunos com o OAMR e proporciona um maior envolvimento destes com os conteúdos apresentados.

4. Possibilitar que diferentes conteúdos didáticos sejam usados com o mesmo OAMR: É comum encontrarmos OAM projetados exclusivamente para atender a um único conteúdo didático. A equipe de desenvolvimento no projeto de um OAMR deve, sempre que possível, elaborar soluções que permitam ao professor posteriormente inserir ou alterar os conteúdos didáticos, permitindo com isto que o mesmo OAM seja utilizado não apenas por grupos diferentes, mas também, pelo mesmo grupo em momentos diferentes.

5. Possibilitar que conteúdos didáticos de um OAMR sejam reutilizados em outras atividades: Outro requisito importante no que concerne aos conteúdos didáticos tem relação com o reaproveitamento destes conteúdos em outras atividades. Os OAMRs devem, sempre que possível, possuir funcionalidades que permitam ao professor reutilizar conteúdos didáticos, seja repetindo integralmente a atividade no qual este conteúdo foi utilizado, seja possibilitando a utilização de partes deste conteúdo em uma nova atividade.

6. Oferecer ferramentas que permitam pesquisa de conteúdos didáticos e referências similares: Segundo Cruz (2017), um dos maiores problemas indicados pelos professores no momento da criação das atividades pedagógicas de um OAMR está na criação e/ou adaptação de conteúdos didáticos a esta nova plataforma.

Neste caso, sempre que possível, devem-se desenvolver funcionalidades que auxiliem o professor nesta tarefa, seja através de tutoriais que facilitem a adaptação do conteúdo, seja indicando fontes nas quais os conteúdos já estejam adaptados para o uso em plataformas mobile.

7. Possuir ferramentas que permitam que usuários com deficiências possam utilizar o OAMR: O projeto de qualquer OAM deve sempre considerar auxiliar alunos e professores com deficiências. Funcionalidades e configurações que permitam que o usuário com deficiência adapte o ambiente as suas necessidades devem, sempre que possível, fazer parte dos requisitos de um OAM.

Muitos dos recursos necessários para promover a inclusão das pessoas com deficiência já estão disponibilizados nos sistemas operacionais dos dispositivos móveis, porém, devem ser desenvolvidas funcionalidades no OAMR para utilizar estes recursos, e sempre que possível, a equipe de desenvolvimento deve validar com professores e alunos se as funcionalidades estão de acordo com a necessidade da pessoa com deficiência.

8. Permitir que o OAMR seja dividido em partes menores e reaproveitáveis em outros contextos: $O$ conceito de reutilização dos OAMR não se refere apenas aos seus conteúdos pedagógicos, mas também ao conjunto de componentes referentes à aplicação (IEEE (2002), retificado em P1484.0012.1-2002 (2010)).

No momento do projeto do OAMR, a equipe de desenvolvimento, sempre que possível, deve optar pelo uso de soluções em componentes ou outras técnicas que permitam que as partes sejam reaproveitadas na criação de outros OAMRs. 
VIII Congresso Brasileiro de Informática na Educação (CBIE 2019)

Anais do XXX Simpósio Brasileiro de Informática na Educação (SBIE 2019)

9. Permitir fácil atualização em segundo plano do OAMR ou de suas partes: Este requisito não se refere apenas à atualização da aplicação, mas também a atualizações relacionadas aos conteúdos didáticos. Ampliar e atualizar os conteúdos didáticos em segundo plano permite que o OAMR ofereça ao aluno sempre novos estímulos educacionais.

10. Facilitar a avaliação de benefícios acadêmicos: Grande parte dos OAMs analisadas para a realização deste estudo ofereciam algum tipo de feedback para os alunos, porém. nenhum apresentou alguma funcionalidade que auxiliasse o professor nas avaliações do grupo ou dos indivíduos.

Os OAMRs devem ser capazes de fornecer não apenas feedbacks para os alunos, mas também, apresentar funcionalidades que permitam que o professor possa acompanhar a evolução acadêmica de suas turmas, e de cada um dos indivíduos por ele acompanhados.

\section{Avaliação dos requisitos em uma aplicação teste}

Após a definição dos requisitos sugeridos para a criação de um OAMR foi desenvolvido uma aplicação teste, visando ratificar se o uso destes requisitos atingiria as expectativas de professores e alunos em relação aos OAM.

A primeira etapa do projeto foi definir que tipo de OAM seria melhor para a proposta. Avaliando os 37 OAMs pesquisados neste estudo, optou-se por desenvolver um jogo de perguntas e respostas (Quizz). Nossa escolha foi ratificada pela afirmação de Freitas et al. (2016) e Rahmalan et al. (2015), quando ambos afirmam que este formato auxilia o professor tanto na apresentação de feedbacks quanto na avaliação do aprendizado.

Outro fator associado a escolha de um jogo de perguntas e respostas como ferramenta para a avaliação dos requisitos é que, segundo Rodriguez et al. (2018), este estilo de jogo auxilia a fixar conceitos e padrões de solução além de facilitar o feedback para os alunos (requisitos 3), e estimular o lado lúdico do aprendizado (requisito 1).

A segunda etapa do desenvolvimento do projeto consistiu em definir o modelo de programação e suas funcionalidades. Foi desenvolvido então uma aplicação híbrida, conforme indicações de Cruz (2017), por permitir que o mesmo código fosse utilizado no ambiente web (módulos do professor e de administração da aplicação) e mobile (módulo do aluno). O desenvolvimento híbrido também facilita a implementação de recursos de acessibilidade mobile indicados pela W3C (requisitos 2,3,7 e 8).

No módulo do professor foram projetadas não apenas funcionalidades que permitem a inserção das questões a serem respondidas pelos alunos, mas também, outras que permitem a consulta e inclusão em banco de dados internos e externos a aplicação, um tutorial para auxiliar na formatação das perguntas e um painel de configurações que permite ao professor definir o layout da aplicação para cada turma (background, cor e estilo de fonte, e textos de orientação das atividades) (requisitos $3,4,5,6$ e 10$)$.

Ainda no módulo do professor foram desenvolvidas duas funcionalidades que permitem que o usuário envie notificações para os grupos de alunos e agendar atualizações de conteúdo didático (requisitos 2 e 9). 
VIII Congresso Brasileiro de Informática na Educação (CBIE 2019)

Anais do XXX Simpósio Brasileiro de Informática na Educação (SBIE 2019) (mobile).

A Figura 1 ilustra as interfaces dos módulos do professor (web) e do aluno
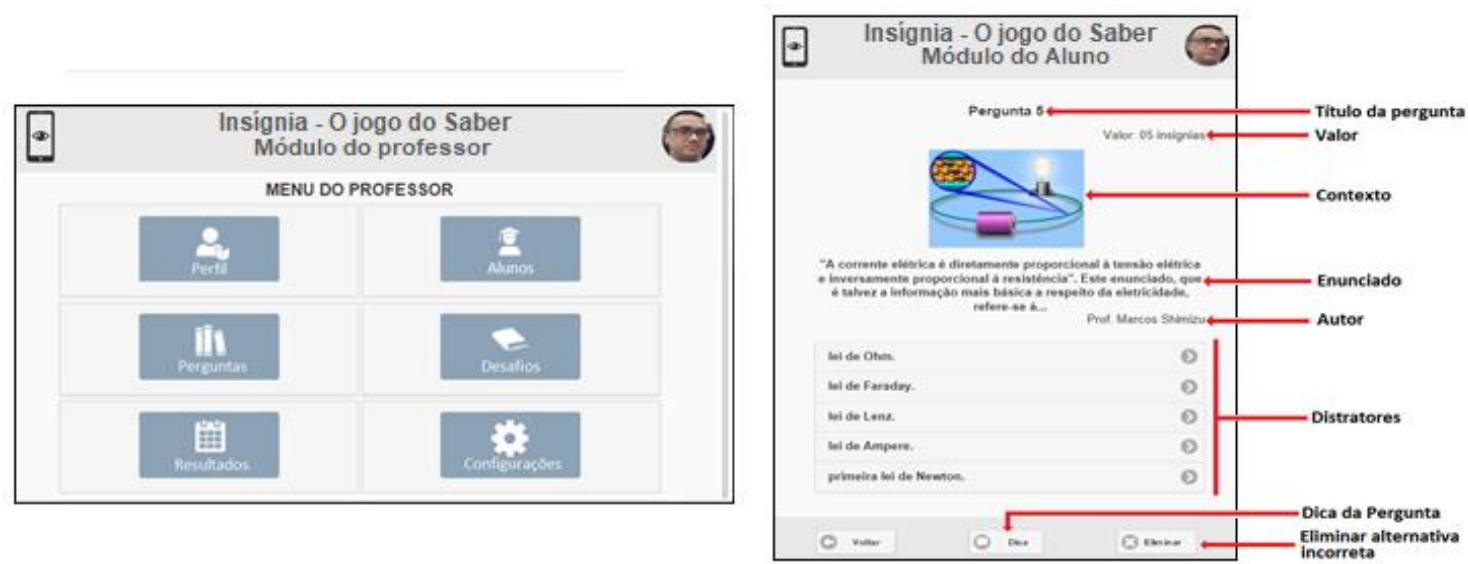

Figura 1 - Interface dos módulos do professor e do aluno da aplicação teste

No módulo do aluno foram desenvolvidas funcionalidades que permitem que ele receba os convites para participar em desafios, personalizar sua identificação nas listas (apelido), receber feedbacks das questões de acordo com seu desempenho, acompanhar sua pontuação em cada desafio e enviar dúvidas para o professor (requisitos 2,3,7 e 10).

\section{Avaliação do OAMR}

Após a conclusão das etapas de desenvolvimento, foram selecionados 166 voluntários (05 professores e 161 alunos) para auxiliar na avaliação do OAMR. Os alunos foram divididos em dois grupos denominados, grupo de teste, aqueles que utilizavam a aplicação como ferramenta de estudo e o grupo de controle, aqueles que respondiam as questões de forma tradicional. A Tabela 1 descreve os grupos de alunos voluntários.

Tabela 1 - Perfil dos grupos de alunos voluntários

\begin{tabular}{|l|l|l|r|r|l|}
\hline Grupo & \multicolumn{1}{c|}{$\begin{array}{c}\text { Perfil } \\
\text { Escolar }\end{array}$} & \multicolumn{1}{c|}{$\begin{array}{c}\text { Nível } \\
\text { Escolar }\end{array}$} & \multicolumn{2}{|c|}{ Voluntários } & \multicolumn{2}{|c|}{ Atividades } \\
\cline { 4 - 6 } Grupo A & Escola Pública & Ensino Fundamental & 13 & 12 & Língua inglesa \\
\hline Grupo B & Escola Particular & Ensino Técnico & 26 & 26 & Redes de Computadores e Arquitetura \\
\hline Grupo C & Escola Pública & Ensino Técnico & 15 & 14 & Logística \\
\hline Grupo D & Escola Particular & Graduação & 22 & 21 & Sistemas de Informação \\
\hline Grupo E & Escola Particular & Graduação & 06 & 06 & Lógica Aplicada a Programação \\
\hline
\end{tabular}

Além da elaboração dos desafios para os alunos, os professores voluntários também ficaram responsáveis por avaliar se a aplicação atendia os requisitos ligados às necessidades pedagógicas. Já os alunos ficaram responsáveis por avaliar os requisitos ligados à usabilidade da aplicação. Ambos responderam a questionários elaborados de acordo com seus perfis.

Ao analisarmos, por exemplo, as respostas fornecidas pelos professores em relação à facilidade do uso das funcionalidades apresentadas no OAMR, 60\% deles consideraram como "Muito simples" e "Adequadas ao contexto". As melhorias, 
VIII Congresso Brasileiro de Informática na Educação (CBIE 2019)

Anais do XXX Simpósio Brasileiro de Informática na Educação (SBIE 2019)

sugeridas pelo mesmo grupo, permeavam principalmente ajustes nos layouts apresentados e a adequação da linguagem utilizada para facilitar a compreensão do professor iniciante.

A avaliação final dos professores, foi considerada positiva em relação aos requisitos, e $100 \%$ deles indicaram que usariam a aplicação teste em suas atividades pedagógicas regulares.

Em relação a avaliação dos alunos sobre o uso da aplicação testes, destaca-se que $80,29 \%$ deles apontaram que a aplicação os estimulou a estudar mais sobre os assuntos propostos e que $74,39 \%$ deles consideraram a usabilidade da aplicação como "Muito boa". A Figura 2 exemplifica as respostas apresentadas pelos alunos.

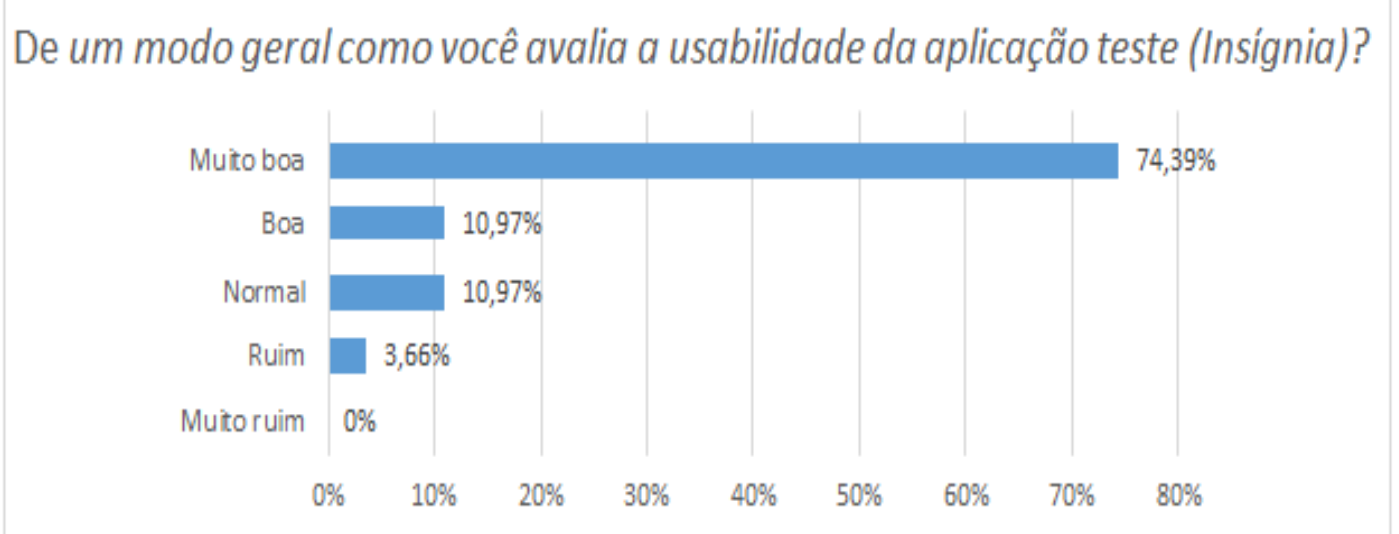

Figura 2 - Opinião dos discentes sobre a aplicação teste

A aplicação teste permitiu também coletar os dados referentes à quantidade de acertos e erros das respostas dos alunos para as atividades didáticas. Os alunos responderam questões de conhecimentos gerais (Desafio A) e conhecimentos específicos das disciplinas (Desafio B). Um resumo dos dados dos grupos de teste e de controle são apresentados na Figura 3 e na Figura 4.

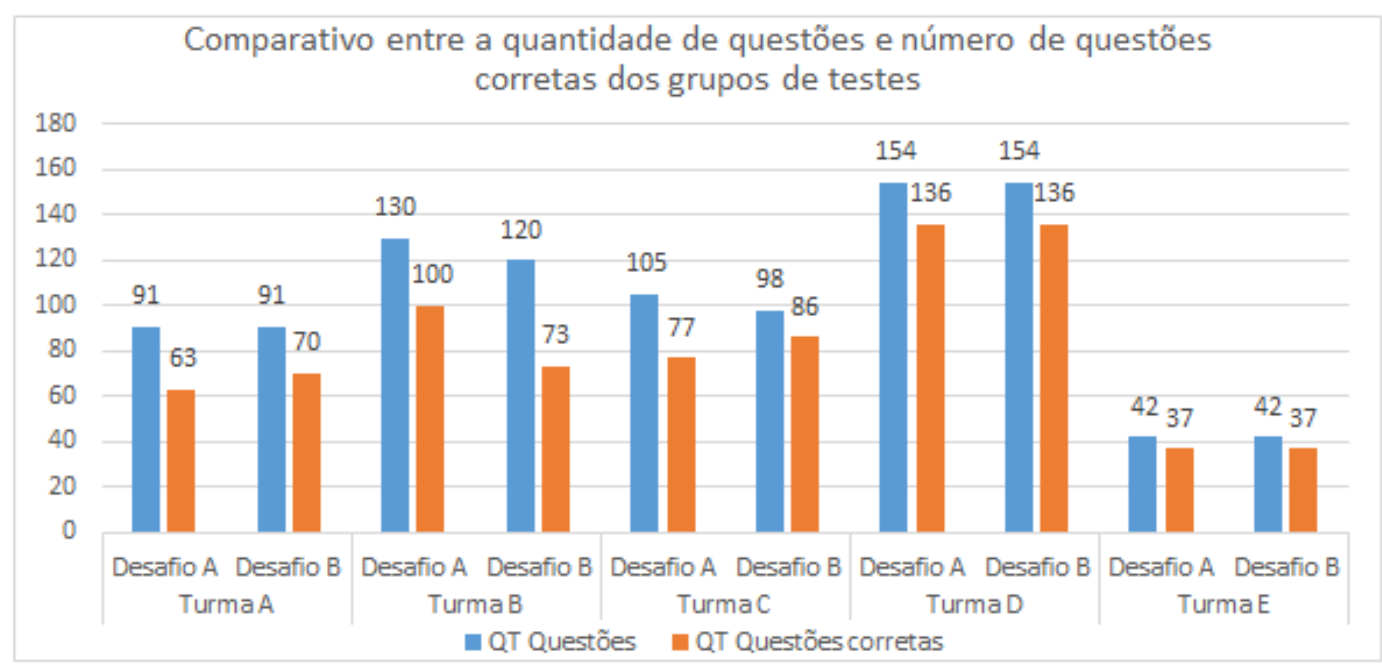

Figura 3 - Número de questões e as respostas corretas dos grupos de teste 
VIII Congresso Brasileiro de Informática na Educação (CBIE 2019)

Anais do XXX Simpósio Brasileiro de Informática na Educação (SBIE 2019)

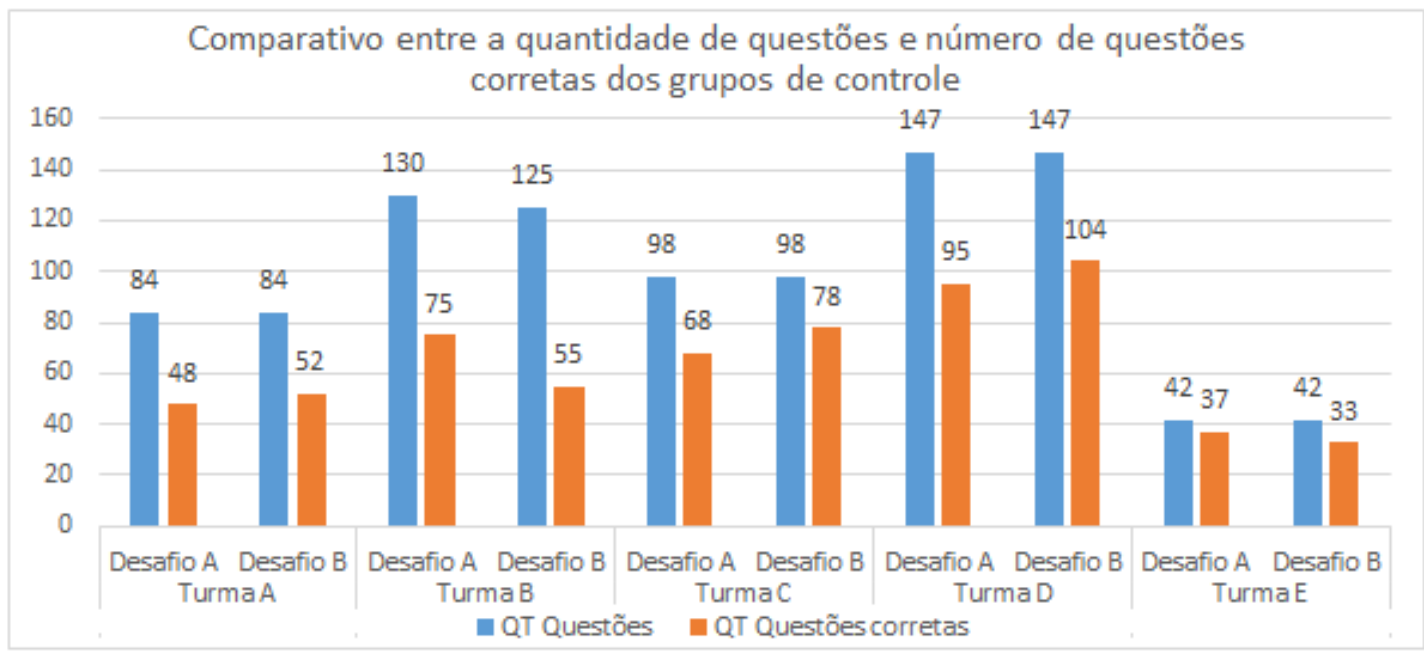

Figura 4 - Número de questões e as respostas corretas dos grupos de controle

Esses dados foram utilizados para analisar de forma quantitativa se o uso do OAMR acarretou algum benefício acadêmico nos grupos de teste com relação aos grupos de controle. Para esses fins foram estabelecidas duas hipóteses (i) Hipótese nula (H0): o uso do OAMR não acarretou benefício acadêmico para os alunos que utilizaram a aplicação; (ii) Hipótese alternativa (HA): o uso do OAMR acarretou benefício acadêmico para os alunos que utilizaram a aplicação.

A Tabela 2 sumariza a média e variância dos grupos analisados assim como os resultados do teste $t$ de Student realizado com um intervalo de confiança de 95\%.

Tabela 2 - Resumo estatístico do grupo de controle e experimental

\begin{tabular}{|l|c|c|c|c|c|c|}
\hline & $\begin{array}{c}\text { Média \% } \\
\text { Acertos }\end{array}$ & Variância & $\begin{array}{c}\mathrm{T} \text { de } \\
\text { Student }(\mathrm{t})\end{array}$ & $\begin{array}{c}\text { Graus de } \\
\text { Liberdade }\end{array}$ & $\mathrm{P}(\mathrm{T}<=\mathrm{t})$ & $\mathrm{t}$ Crítico \\
\cline { 1 - 6 } Grupos de Controle & 66,9 & 163,211 & & & & \\
\cline { 1 - 6 } Grupos Experimentais & 79,7 & 96,455 & -8.6754 & 18 & 0,0217 & 2,1009 \\
\hline
\end{tabular}

Dado que foi obtido um valor $p$ de 0,0217 que é inferior ao nível de significância $(0,05)$, o teste indica a rejeição da H0. Portanto, o uso do OAMR acarretou benefício acadêmico para os alunos que utilizaram a aplicação.

\section{Considerações finais}

O trabalho aqui apresentado começou relatando os conceitos de OAMR e reportando uma pesquisa sobre o tema nos anais de eventos ligados ao uso de tecnologia na educação. Como resultado da análise desse material foram definidos 10 requisitos para auxiliar professores, pesquisadores e desenvolvedores na criação de OAMRs.

Para avaliar se estes requisitos eram factíveis para o uso no processo de criação de um OAMR foi desenvolvida uma aplicação teste e com a ajuda de 166 voluntários, esta aplicação foi analisada visando saber se atendia as necessidades de professores e alunos.

Como resultado da avaliação foi possível observar que os requisitos apresentados neste artigo se mostraram factíveis, e resultaram em uma aplicação teste 
VIII Congresso Brasileiro de Informática na Educação (CBIE 2019)

Anais do XXX Simpósio Brasileiro de Informática na Educação (SBIE 2019)

que não só apresentou avaliação positiva por parte de professores e alunos, mas também, representou uma melhora no nível de aprendizagem dos alunos.

Como trabalhos futuros iremos estimular o desenvolvimento de outras aplicações usando como referência os requisitos aqui indicados, para observar se os resultados obtidos por estas aplicações serão semelhantes ao obtidos inicialmente. Também pretendemos realizar um estudo no qual a aplicação teste seja usada como ferramenta de auxílio ao professor durante um período letivo completo, o que permitirá assim ratificar os dados apresentados neste estudo.

\section{Referências:}

Brasil, S.B., Santos, Be. P. \& Ferenhof, H. A. (2018). Mobile learning: Um estudo exploratório sobre aprendizagem com mobilidade no brasil. IJKEM - International Journal of Knowledge Engineering and Management, Universidade de Santa Catarina, vol.7, num. 19, Santa Catarina.

Cruz (2017). Declaração de Princípios para o Desenvolvimento de Objetos de Aprendizagem Reutilizáveis para Dispositivos Móveis. Dissertação Programa de Mestrado do Centro Universitário UNIFACCAMP, URL: http://www.cc.faccamp. br/Dissertacoes/JoaoRobertoUrsinoCruz.pdf, acessado em 22/04/2019

Freitas, S., Lima, T., Canedo, E., \& Costa, R. L. (2016). Gamificação e avaliação do engajamento dos estudantes em uma disciplina técnica de curso de graduação. XXVII Simpósio Brasileiro de Informática em Educação 2016.

IEEE (2010). P1484.12.1-2002 / COR 1_D13, Jan 2010 - Projeto de Norma IEEE para Metadados de Objetos de Aprendizagem - Corrigenda 1: Corrigenda para 1484.12.1 LOM (Metadados de Objetos de Aprendizagem), ISBN eletrônico: 978-1-50442959-7, URL: https://ieeexplore.ieee.org/servlet/opac?punumber=5445241

Pereira, L. A., Porto, F. A., \& Melo, R. N. (2003). Objetos de Aprendizado Reutilizáveis (RLOs): Conceitos, Padronização, Uso e Armazenamento. PUC - Rio.

Rahmalan, H., Abidin, Z. Z., Abas, Z. A., Rahman, A. F., Shibghatullah, A. S., \& RhiYong, O. (2015). Prototype deployment of examination and quiz system (EQs) with outcome based education in networking system. Proceedings of the 2015 IEEE International Conference Communication, Networks and Satellite (COMNESTAT).

Rodriguez, C. L.,Rocha, R. V., Goya, D.H., Venero, M.L.F., Zampirolli, F. (2018). Critérios para inserção de estratégias cognitivas e metacognitivas no desenvolvimento de lógica de programação em ambientes virtuais de aprendizagem. XXIX Simpósio Brasileiro de Informática na Educação (SBIE 2018)

Silva, L.C.N. (2012). MobiLE - Um ambiente Multiagente de Aprendizagem Móvel para Apoiar a Recomendação Ubíqua de Objetos de Aprendizagem. Programa de Mestrado em Ciências da Computação da Universidade Federal Rural do Semiárido. Mossoró.

Sommerville, I. (2011). Engenharia de Software. ed. 9a , ISBN 9788579361081. São Paulo, Pearson.

Wiley, D. (2000). Learning Object Design and Sequenceing Theory. 2000. Tese (Doutorado em Filosofia). Brigham Young University, Provo, 2000. 